Pacific Journal of Mathematics

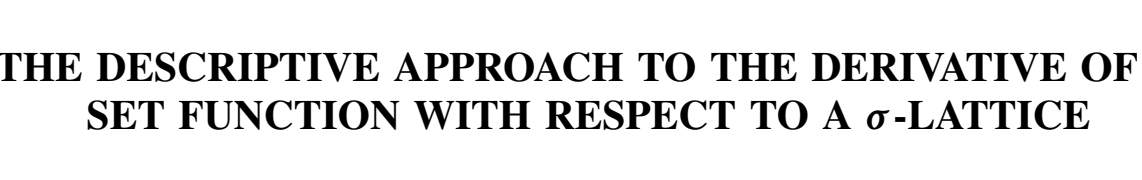




\title{
THE DESCRIPTIVE APPROACH TO THE DERIVATIVE OF A SET FUNCTION WITH RESPECT TO A $\sigma$-LATTICE
}

\author{
S. JOHANSEN
}

\begin{abstract}
This paper contains a definition and a construction of a Radon-Nikodym derivative of a $\sigma$-additive set function with respect to a measure on a $\sigma$-lattice, that is, a family of sets closed under countable unions and countable intersections. This derivative is characterized in terms of its indefinite integral, and it is shown how the conditional expectation of an integrable random variable with respect to a $\sigma$-lattice, as defined by Brunk, can be obtained as a Radon-Nikodym derivative of the set function determined by the indefinite integral of the random variable.
\end{abstract}

The so-called descriptive approach to the theory of Radon-Nikodym derivatives tells us that if $\varphi$ is a finite $\sigma$-additive set function defined on a $\sigma$-field $\mathscr{A}$ and if $\mu$ is a positive measure, then there exists an $\mathscr{A}$ measurable function $f$ such that for all $B \in \mathscr{A}$ and $C \in \mathscr{A}$ we have

$$
\varphi(B \cap[f<a]) \leqq a \mu(B \cap[f<a]), \quad a \in R,
$$

and

$$
\varphi(C \cap[f>b]) \geqq b \mu(C \cap[f>b]), \quad b \in R .
$$

The inequalities easily imply that the function is unique and finite 8 almost surely.

It turns out that if $\mathscr{C}$ is a $\sigma$-lattice, and if $\varphi$ is a finite $\sigma$-additive set function defined on sets of the form $B \cap C, B \in \mathscr{M}, C \in \mathscr{C l}^{c}$, then exactly the same construction can be used to give a function, which is $\mathscr{C l}$ measurable and satisfies the two inequalities above with $B \in \mathscr{C l}$ and $C \in \mathscr{C l}^{c}=\left\{A \mid A^{c} \in \mathscr{C}\right\}$. The main fact we need is, that the Hahn decomposition remains valid for the set function $\varphi$. This was remarked already in [3], where a similar idea was used to discuss the conditional expectation on $n$-dimensional euclidian space. By means of this approach it is easy to obtain a proof of the (closed) martingale convergence theorem for $\sigma$-lattices and to identify the limit function; in fact the proof by Andersen and Jessen [1] can be applied without any change. See also [2] and [5].

1. The Hahn decomposition. Let $\Omega$ be an abstract space, and let $\mathscr{A}$ be a $\sigma$-field of subsets of $\Omega$. Let $\mathscr{C}$ be a $\sigma$-lattice of sets from $\mathscr{A}$, such that $\varnothing \in \mathscr{C}, \Omega \in \mathscr{C}$. Define $\mathscr{F}=\{A: A=B \cap C, B \in \mathscr{K}$, 
$\left.C \in \mathscr{M}^{c}\right\}$. Let $\nu$ be a $\sigma$-additive set function defined on $\mathscr{F}$ with values in $[-\infty,+\infty]$.

A set $B \in \mathscr{C l}$ is called positive, if for all $C \in \mathscr{C l}^{c}$, we have $\nu(B \cap C) \geqq 0$. A set $C \in \mathscr{K}^{c}$ is called negative if for all $B \in \mathscr{C l}$ we have $\nu(B \cap C) \leqq 0$. Let $\mathscr{P}$ be the family of positive sets and let $\mathscr{N}$ be the family of negative sets, then $\varnothing \in \mathscr{N} \cap \mathscr{P}$, and $\mathscr{P}$ and $\mathscr{N}$ are closed under countable unions. A set $C_{0} \in \mathscr{N}$ is called minimal if $\nu C_{0}=$ $\inf _{\sigma \in \mathscr{N}} \nu C$ and $B_{0} \in \mathscr{C}$ is called maximal if $\nu B_{0}=\sup _{B \in \mathscr{O}} \nu B$. It is easily seen that there exists as well minimal as maximal sets. We now need the following theorem.

Theorem 1. If $\nu<+\infty$ and if $A$ is a maximal set then $A^{c}$ is negative, and in fact minimal.

CoRollary (Jordan-Hahn decomposition). There exists a maximal set $A^{+} \in \mathscr{L l}$ and a minimal set $A^{-} \in \mathscr{l}^{c}$ such that $A^{-}=\left(A^{+}\right)^{c}$ and

$$
\begin{array}{ll}
\nu\left(A^{+} \cap C\right) \geqq 0, & C \in \mathscr{C}^{c}, \\
\nu\left(A^{-} \cap B\right) \leqq 0, & B \in \mathscr{C l} .
\end{array}
$$

Proof of Theorem 1. The proof which is given here follows in details the proof given in Halmos [4] (p. 122), for the ordinary Hahn decomposition. Let $A$ be a maximal set, then $\nu(A)<+\infty$ and we assume that $A^{c}$ is not in $\mathscr{N}$. Then there exists a set $B_{0} \in \mathscr{C l}$ such that $\nu\left(A^{c} \cap B_{0}\right)>0$. There must be sets $C \in \mathscr{l}^{c}$ such that $\nu\left(A^{c} \cap B_{0} \cap C\right)<0$, since otherwise $A \cup B_{0}$ would be $\in \mathscr{P}$ and have measure larger than $A$. Let therefore $k_{i}$ be the smallest integer such that there exists $C_{1} \in \mathscr{M}^{c}$ with the property $\nu\left(A^{c} \cap B_{0} \cap C_{1}\right) \leqq-1 / k_{1}$. Having defined $C_{i}$, and $B_{i}=C_{i}^{c} i=1, \cdots, n-1$, we still have

$$
\nu\left(A^{c} \cap B_{0} \cap B_{1} \cap \cdots \cap B_{n-1}\right)>0,
$$

but again we can find the smallest integer $k_{n}$ such that there exists $C_{n}$ with the property $\nu\left(A^{c} \cap B_{0} \cap B_{1} \cap \cdots \cap B_{n-1} \cap C_{n}\right) \leqq-1 / k_{n}$. In this way we construct an infinite sequence $C_{n}, n \geqq 1$.

From

$$
\begin{aligned}
-\infty & <\nu\left(A^{c} \cap B_{0} \cap \bigcup_{n=1}^{\infty} C_{n}\right)=\sum_{n=1}^{\infty} \nu\left(A^{c} \cap B_{0} \cap B_{1} \cap \cdots \cap B_{n-1} \cap C_{n}\right) \\
& \leqq \sum_{n=1}^{\infty}-1 / k_{n}
\end{aligned}
$$

we get that $k_{n} \rightarrow \infty$. Let $F=\bigcap_{n=0}^{\infty} B_{n}$. To show that $F \cup A$ is positive we evaluate for $C \in \mathscr{M}^{\circ}$ 


$$
\nu((F \cup A) \cap C)=\nu(A \cap C)+\nu\left(A^{c} \cap F \cap C\right),
$$

but $\nu(A \cap C) \geqq 0$ and

$$
\nu\left(A^{c} \cap F \cap C\right)=\lim _{n \rightarrow \infty} \nu\left(A^{c} \cap C \cap \bigcap_{i=0}^{n-1} B_{i}\right) \geqq \lim _{n \rightarrow \infty}\left(-1 /\left(k_{n}-1\right)\right)=0 .
$$

Finally

$$
\nu\left(A^{c} \cap F\right)=\nu\left(A^{c} \cap B_{0} \cap \bigcap_{n=1}^{\infty} B_{n}\right)=\nu\left(A^{c} \cap B_{0}\right)-\nu\left(A^{c} \cap B_{0} \cap \bigcup_{n=1}^{\infty} C_{n}\right)>0
$$

which implies that $A \cup F \in \mathscr{P}$ and $\nu(A \cup F)>\nu(A)$, contradicting the maximality of $A$. Hence it is established that $A^{c} \in \mathscr{N}$ and it is easily seen that $A^{c}$ is minimal.

2. The derivative. Let there be given a set function $\varphi$ on $\mathscr{F}$ such that $\varphi$ is finite, $\varphi$ is $\sigma$-additive and such that $\varphi(C) \geqq 0, C \in \mathbb{C l}^{c}$. Let $\mu$ be a measure on $\mathscr{F}$, i.e. $\mu(A) \geqq 0, A \in \mathscr{F}, \mu \sigma$-additive.

For each $a \in R$ let $\nu_{a}=\varphi-a \mu$. Then we have for $a>0$ that $-\infty \leqq \nu_{a}<+\infty$, and if we define a positive maximal set $A_{a}^{+}$then $A_{a}^{-}=\left(A_{a}^{+}\right)^{c}$ is negative for $\nu_{a}$ by Theorem 1 . If $a \leqq 0$ we can choose $A_{a}^{+}=\Omega, A_{a}^{-}=\varnothing$. Now define a function $f$ in the following way:

$$
f(\omega)=\sup \left\{r \mid r \text { rational, } \omega \in A_{r}^{+}\right\} \text {. }
$$

Clearly $f$ is defined on all of $\Omega$ and $f \geqq 0$. Further $\lfloor f>a]=$ $\mathrm{U}_{r>a} A_{r}^{+}$if $a \geqq 0$ and $[f>a]=\Omega$ if $a<0$, which proves that $f$ is measurable $\mathscr{C}$.

The sets $A_{a}^{+}$are not in general decreasing in $a$ but we could choose them to be as the following proposition shows:

Proposition 1. For all $a$ the set $[f>a]$ is a positive set for $\nu_{a}$, while for $a \neq 0$ it is maximal.

Proof. For $a<0$ the assertions are trivial. Let $a \geqq 0$, then for $r>a, A_{r}^{+}$is positive for $\nu_{a}$, since

$$
\nu_{a}\left(A_{r}^{+} \cap C\right) \geqq \nu_{r}\left(A_{r}^{+} \cap C\right) \geqq 0, \quad C \in \mathscr{C}^{c} .
$$

But the system of positive sets is closed under countable unions, which proves the first statement.

Now let $a>0$, to see that $[f>a]=\bigcup_{r>a} A_{r}^{+}$is maximal, we first compare $\bigcup_{i=1}^{n} A_{r_{i}}^{+}$with $A_{q_{n}}^{+}$, where $r_{i}$ is an enumeration of the rationals greater than $a$ and $q_{n}=\min _{1 \leqq i \leqq n} r_{i} \downarrow a$ for $n \rightarrow \infty$. From the inequality

$$
0 \leqq \nu_{r_{i}}\left(A_{r_{i}}^{+} \cap A_{q_{n}}^{-}\right) \leqq \nu_{q_{n}}\left(A_{r_{i}}^{+} \cap A_{q_{n}}^{-}\right) \leqq 0
$$


we get, that $\mu\left(A_{r_{i}}^{+} \cap A_{q_{n}}^{-}\right)=0 i=1, \cdots, n$, and hence that

$$
\mu\left(\bigcup_{i=1}^{n} A_{r_{i}}^{+} \cap A_{q_{n}}^{-}\right)=0 .
$$

Therefore

$$
0 \leqq \nu_{a}\left(\bigcup_{i=1}^{n} A_{r_{i}}^{+} \cap A_{q_{n}}^{-}\right)=\nu_{q_{n}}\left(\bigcup_{i=1}^{n} A_{r_{i}}^{+} \cap A_{q_{n}}^{-}\right) \leqq 0
$$

which yields

$$
\nu_{a}\left(A_{q_{n}}^{+}\right)=\nu_{a}\left(\bigcup_{i=1}^{n} A_{r_{i}}^{+}\right) .
$$

We now compare $A_{q_{n}}^{+}$with $A_{a}^{+}$as follows:

$$
0 \leqq \nu_{q_{n}}\left(A_{a}^{-} \cap A_{q_{n}}^{+}\right) \leqq \nu_{a}\left(A_{a}^{-} \cap A_{q_{n}}^{+}\right) \leqq 0
$$

and $0 \leqq \nu_{a}\left(A_{a}^{+}\right)$which implies that

$$
\mu\left(A_{a}^{+} \cap A_{q_{n}}^{-}\right) \leqq \mu\left(A_{a}^{+}\right) \leqq \frac{1}{a} \varphi\left(A_{a}^{+}\right)=c<\infty .
$$

Finally we have

$$
\begin{aligned}
0 \leqq \nu_{a}\left(A_{a}^{+} \cap A_{q_{n}}^{-}\right) & =\nu_{q_{n}}\left(A_{a}^{+} \cap A_{q_{n}}^{-}\right)+\left(q_{n}-a\right) \mu\left(A_{a}^{+} \cap A_{q_{n}}^{-}\right) \\
& \leqq\left(q_{n}-a\right) c .
\end{aligned}
$$

Combining the evaluations (1), (2) and (3) we get

$$
\nu_{a}\left(A_{a}^{+}\right) \geqq \nu_{a}\left(\bigcup_{i=1}^{n} A_{r_{i}}^{+}\right)=\nu_{a}\left(A_{q_{n}}^{+}\right) \geqq \nu_{a}\left(A_{a}^{+}\right)-\left(q_{n}-a\right) c .
$$

Letting $n \rightarrow \infty$ we get, that $q_{n} \downarrow \alpha$ and

$$
\nu_{a}\left(A_{a}^{+}\right)=\nu_{a}\left(\bigcup_{r>a} A_{r}^{+}\right)=\nu_{a}[f>a],
$$

which proves that $[f>a]$ is not only positive but also maximal for $\nu_{a}$.

DeFINITION 1. If $\varphi$ is a finite $\sigma$-additive set function on $\mathscr{F}$ and if $\mu$ is a measure on $\mathscr{F}$, then we call $f$ a derivative of $\varphi$ with respect to $\mu$ on $\mathscr{C}$ if $f$ is an extended real valued function defined on $\Omega$, and if

(1) $f$ measurable $\mathscr{C}$,

(2) $\varphi(B \cap[f<b]) \leqq b \mu(B \cap[f<b]), B \in \mathscr{L}, b \in R$,

(3) $\varphi(C \cap[f>a]) \geqq a \mu(C \cap[f>a]), C \in \mathbb{L}^{c}, a \in R$.

THEOREM 2. If $\varphi$ is a finite $\sigma$-additive set function defined on 
$\mathscr{F}$ and if $\mu$ is a measure on $\mathscr{F}$, then there exists a derivative $f$ of $\phi$ with respect to $\mu$ on $\mathscr{C l}$. Any derivative is finite $\mu$ almost surely, and if $g$ and $h$ are two derivatives then $\mu[h<a<b<g]=0$, i.e. if $\mu$ is defined on $\mathscr{A}$ then $\mu[h \neq g]=0$.

Proof. According to Theorem 1 we can find a maximal set $A_{0}^{+}$ and a minimal set $A_{0}^{-}$such that $A_{0}^{-}=\left(A_{0}^{+}\right)^{c}$. We define $\varphi^{+}$and $\mu^{+}$as the contraction of $\varphi$ and $\mu$ to $A_{0}^{+}$, and we define $\varphi^{-}$and $\mu^{-}$as the contraction of $-\varphi$ and $\mu$ to $A_{0}^{-}$. Then we can construct a function $f^{+}$defined on $A_{0}^{+}$, and measurable $\mathscr{C l}$ by means of $\varphi^{+}$and $\mu^{+}$as indicated in the beginning of this section. We also construct $f^{-}$from $\mathcal{P}^{-}$and $\mu^{-}$such that $f^{-}$is defined on $A_{0}^{-}$and measurable $\mathscr{C}^{c}$.

Now define $f$ as follows:

$$
f(\omega)=\left\{\begin{aligned}
f^{+}(\omega), & \omega \in A_{0}^{+}, \\
-f^{-}(\omega), & \omega \in A_{0}^{-} .
\end{aligned}\right.
$$

We then have

$$
[f>a]=\left\{\begin{array}{l}
{\left[f^{+}>a\right], \quad a \geqq 0,} \\
A_{0}^{+} \cup\left[f^{-}<-a\right], \quad a<0,
\end{array}\right.
$$

which proves that $f$ is measurable $\mathscr{l}$.

To prove (2) and (3) of Definition 1, we remark that for $a \geqq 0$ we have $[f>a]=\left[f^{+}>a\right]$ which by Proposition 1 is positive for $\phi^{+}-a \mu^{+}$, hence

$$
\begin{aligned}
\varphi(C \cap[f>a]) & =\varphi^{\dagger}\left(C \cap\left[f^{+}>a\right]\right) \geqq a \mu^{+}\left(C \cap\left[f^{+}>a\right]\right) \\
& =a \mu(C \cap[f>a]) .
\end{aligned}
$$

For $a>0$, we have that $[f>a]=\left[f^{+}>a\right]$ is maximal for $\varphi^{+}-a \mu^{+}$ and then by Theorem $1\left[f^{+} \leqq a\right]$ is negative for $\varphi^{+}-a \mu^{+}$, hence

$$
\begin{aligned}
\varphi(B \cap[f \leqq a]) & =\varphi\left(B \cap A_{0}^{-}\right)+\varphi\left(B \cap A_{0}^{+} \cap\left[f^{+} \leqq a\right]\right) \\
& \leqq \varphi^{+}\left(B \cap\left[f^{+} \leqq a\right]\right) \leqq a \mu^{+}\left(B \cap\left[f^{+} \leqq \alpha\right]\right) \\
& =a \mu\left(B \cap A_{0}^{+} \cap[f \leqq a]\right) \leqq a \mu(B \cap[f \leqq a]) .
\end{aligned}
$$

For $a \uparrow b$ we get (2) for $b>0$. Similarly we prove (2) for $b \leqq 0$ and (3) for $a<0$, by means of the properties of $f^{-}$. This proves the existence of a derivative. To see, that any derivative is finite we note, that for $a>0$ we have

$$
a \mu[|f|>a] \leqq \varphi[f>a]-\varphi[f<-a] \leqq \varphi\left(A_{0}^{+}\right)-\varphi\left(A_{0}^{-}\right)<+\infty,
$$

and for $a \rightarrow \infty$ we get the result.

Finally, let $h$ and $g$ be any two derivatives, then 


$$
b \mu[h<a<b<g] \leqq \varphi[h<a<b<g] \leqq a \mu[h<a<b<g],
$$

which implies, that all terms are 0 .

The following theorem relates the function $\varphi$ to the indefinite integral of its derivative. Let $\varphi_{c}, \varphi_{s}^{+}$and $\varphi_{s}^{-}$be defined as follows:

$$
\begin{array}{ll}
\varphi_{c}(A)=\varphi(A \cap[-\infty<f<0])+\varphi(A \cap[0<f<+\infty]), & A \in \mathscr{F}, \\
\varphi_{s}^{+}(A)=\varphi(A \cap[f=+\infty]) & , A \in \mathscr{F}, \\
\varphi_{s}^{-}(A)=-\varphi(A \cap[f=-\infty]) & , A \in \mathscr{F} .
\end{array}
$$

Theorem 3. If $f$ is a derivative of $\phi$ with respect to $\mu$ on $\mathscr{M}$, where $\mu$ is a measure on $\mathscr{A}$, then $f$ is integrable and

(1) $\varphi_{c}(C) \geqq \int_{0} f d \mu, C \in \mathscr{C}^{c}$,

(2) $\varphi_{c}(B) \geqq \int_{B} f d \mu, B \in \mathscr{L}$,

(3) $\varphi_{\mathrm{c}}[a<f<b]=\int_{[a<f<b]} f d \mu, a \in R, b \in R$,

(4) $\varphi_{s}^{+}(C) \geqq 0, C \in \mathscr{L}^{c}, \varphi_{s}^{-}(B) \geqq 0, B \in \mathscr{l}$.

Proof. Define for $k \geqq 0, \rho<1$ and $b>0 A_{k}=\left[b \rho^{k+1} \leqq f<b \rho^{k}\right]$. Then

$$
\begin{aligned}
\varphi_{c}(C \cap[0<f<b]) & =\sum_{k=0}^{\infty} \varphi\left(C \cap A_{k}\right) \geqq \sum_{k=0}^{\infty} \rho^{k+1} b \mu\left(C \cap A_{k}\right) \\
& \geqq \rho \sum_{k=0}^{\infty} \int_{\sigma \cap A_{k}} f d \mu=\rho \int_{o \cap[0<f<b]} f d \mu .
\end{aligned}
$$

For $\rho \uparrow 1$ we get

$$
\varphi_{c}(C \cap[0<f<b]) \geqq \int_{o \cap[0<f<b]} f d \mu .
$$

In the same way we prove

$$
\begin{gathered}
\varphi_{c}(C \cap[-b<f<0]) \geqq \int_{O \cap[-b<f<0]} f d \mu, \\
\varphi_{c}(B \cap[0<f<b]) \leqq \int_{B \cap[0<f<b]} f d \mu, \\
\varphi_{c}(B \cap[-b<f<0]) \leqq \int_{B \cap[-b<f<0]} f d \mu .
\end{gathered}
$$

From these inequalities follows the integrability of $f$, and the assertions (1), (2) and (3) of the theorem.

Finally we have

$$
\varphi(C \cap[f>a]) \geqq a \mu(C \cap[f>a]) \geqq 0, \quad a>0,
$$


and

$$
\varphi(B \cap[f<-a]) \leqq-a \mu(B \cap[f<-a]) \leqq 0, \quad a>0,
$$

and for $a \rightarrow \infty$ we get (4).

Notice, that nothing is said about $\varphi$ on the set $[f=0]$, so that the 4 results will not characterize the derivative. ( $f \equiv 0$ will always satisfy (1) - (4).) If however $\mu<+\infty$, then Theorem 4 below tells us, that $\varphi(B \cap[f=0]) \leqq 0, B \in \mathscr{C l}$ and $\varphi(C \cap[f=0]) \geqq 0, C \in \mathscr{C}^{c}$, hence we can replace $\phi_{c}$ of Theorem 3 by $\varphi$ contracted to the set $[|f|<+\infty]$. In this case it is easily seen that if $f$ is integrable then (1) through (4) implies (2) and (3) of Definition 1, so that we can characterize the derivative in terms of its indefinite integral.

Theorem 4. If $\mu<+\infty$ we can characterize a derivative of $\varphi$ with respect to $\mu$ on $\mathbb{C l}$ as a function $f$ defined on $\Omega$ such that

(1) $f$ measurable $\mathscr{L}$,

(2) $\varphi(B \cap[f \leqq a]) \leqq a \mu(B \cap[f \leqq a]), B \in \mathbb{l}, a \in R$,

(3) $\varphi(C \cap[f \geqq b]) \geqq b \mu(C \cap[f \geqq b]), C \in \mathscr{K}^{c}, b \in R$.

Proof. We just apply facts of the type

$$
[f>a] \downarrow[f \geqq b], \quad a \uparrow b
$$

and

$$
\lfloor f \geqq a] \uparrow[f>b], \quad a \downarrow b .
$$

3. The minimizing property of the derivative. We want to prove, that the derivative constructed here has the minimizing property, which Brunk has used as the definition. Let therefore $X$ be a real valued measurable function on $(\Omega, \mathscr{A}, \mu)$, such that $\int|X| d \mu<+\infty$. Define $\varphi$ as $\varphi(A)=\int_{A} X d \mu, A \in \mathscr{A}$. We can now apply the preceding theorems to the setup $(\Omega, \mathscr{F}, \mu, \varphi)$. We denote a derivative of $\varphi$ with respect to $\mu$ on $\mathscr{l}$ by $E(X \mid \mathscr{C})$, which we shall call the conditional expectation of $X$ given $\mathscr{C}$. Notice, that $\mu$ dominates $\varphi$, so that any $\mathscr{C l}$ measurable function, which is $\mu$ almost surely equal to a derivative, is itself a derivative. It is natural in this case to denote by $E(X \mid \mathscr{C l})$ the $\mu$-equivalence class which contains the derivative defined earlier. We have thus defined the conditional expectation as a projection in $L_{1}=L_{1}(\Omega, \mathscr{A}, \mu)$. We want to prove the following theorem (see Brunk [2], Theorem 3.1.)

Theorem 5. If $X \in L_{1} \cap L_{2}(\Omega, \mathscr{A}, \mu)$, then $Y=E(X \mid \mathscr{C l})$ is an Ml-measurable function defined on $\Omega$ which is characterized by the 
following conditions:

(1) $Y \in L_{1} \cap L_{2}$,

(2) $\int X Z d \mu \leqq \int Y Z d \mu, Z$ measurable $\mathscr{l}, Z \in L_{1} \cap L_{2}$,

( 3 ) $\int X Z d \mu \geqq \int Y Z d \mu, Z$ measurable $-\mathscr{l}^{c}, Z \in L_{1} \cap L_{2}$,

(4) $\int X Y d \mu=\int Y^{2} d \mu$.

Furthermore $Y$ minimizes the expression $\int(X-Z)^{2} d \mu$ among all $Z \in L_{1} \cap L_{2}$ which are measurable $/$.

Proof. It is easily seen by writing $Z=Z^{+}-Z^{-}$, that it is enough to prove (2) and (3) for $Z \geqq 0$. It is also enough to prove the theorem for $\varphi$ such that $\varphi(C) \geqq 0, C \in \mathbb{C}^{c}$, that is for $Y \geqq 0$.

It follows from Theorem 3 that $Y \in L_{1}$. Hence if $X \in L_{2}$, we have $Y_{2}=E\left(X^{2} \mid \mathscr{C l}\right) \in L_{1}$.

We now prove that

$$
\mu\left[Y^{2}>Y_{2}\right]=0 \text {. }
$$

This inequality clearly implies, that $Y \in L_{2}$. To prove (8) it is enough to see, that for all $a \geqq 0$ and $b \geqq 0$ the set $A=\left[Y>a>b>\sqrt{Y_{2}}\right]$ has $\mu$ measure 0 . We have from the definition of $Y$ and $Y_{2}$, that $[Y>a] \in \mathscr{C l}$ and $\left[\sqrt{Y_{2}}<b\right]=\left[Y_{2}<b^{2}\right] \in \mathscr{C l}^{c}$. Hence

$$
\int_{A} X d \mu \geqq a \mu(A) \text {, }
$$

and

$$
\int_{A} X^{2} d \mu \leqq b^{2} \mu(A)
$$

But we also have

$$
\left(\int_{A} X d \mu\right)^{2} \leqq \int_{A} X^{2} d \mu \int_{A} 1 d \mu=\int_{A} X^{2} d \mu \mu(A)
$$

which imply, that $\mu(A)=0$.

Now let $Z \geqq 0, Z \in L_{1} \cap L_{2}$, and $Z$ measurable $\mathscr{C l}$. For $a>1$ we define

$$
Z_{a}=\sum_{n=-\infty}^{\infty}\left(a^{n+1}-a^{n}\right) I\left[Z>a^{n+1}\right] .
$$

We have $Z_{a} \leqq Z \leqq a Z_{a}$ and $\left|Z_{a}-Z\right| \leqq|Z|(1-1 / a)$. Applying the definition of $Y$ we easily get

$$
\int_{B \cap[Z>0] \cap[X<b]} X Z_{a} d \mu \leqq b \int_{B \cap[Z>0] \cap[Y<b]} Z_{a} d \mu, \quad B \in \mathscr{C l}, \quad b \in R .
$$


For $a \downarrow 1$ we obtain

$$
\int_{B \cap[Y<b]} X Z d \mu \leqq b \int_{B \cap[Y<b]} Z d \mu, \quad B \in \mathscr{C l}, \quad b \in R .
$$

Since both terms are finite we can let $b \downarrow 0$ and we get for $B=$ $[Y \geqq 0]$, that

$$
\int_{[Y=0]} X Z d \mu \leqq 0
$$
that

If we choose $\rho>1, b=\rho^{n+1}, B=\left[Y \geqq \rho^{n}\right]$, then we get from (9)

$$
\int_{[Y>0]} X Z d \mu \leqq \int_{[Y>0]} Y Z d \mu .
$$

Now (10) and (11) implies (2), and (3) is proved analogously.

From Theorem 3 we get, that

$$
\int_{\left[\rho^{n} \leqq Y<\rho^{n+1}\right]} X d \mu=\int_{\left[\rho^{n} \leqq Y<\rho^{n+1}\right]} Y d \mu .
$$

By multiplication with $\rho^{n}$ and summation over $n$ we get for $\rho \downarrow 1$ that (4) holds.

The last assertion now follows from

$$
\begin{aligned}
\int(X-Z)^{2} d \mu= & \int(X-Y)^{2} d \mu \\
& +\int(Y-Z)^{2} d \mu+2 \int(X-Y)(Y-Z) d \mu,
\end{aligned}
$$

since

$$
\int(X-Y)(Y-Z) d \mu=-\int(X-Y) Z d \mu \geqq 0 .
$$

Further let $Z_{1}$ be any $\mathscr{C}$ measurable function satisfying (1) through (4) of Theorem 5 , then $Z_{1}$ minimizes $\int(X-Z)^{2} d \mu$ and from (12) we get for $Z=Z_{1}$, that $Z_{1}=E(X \mid \mathscr{M})$ and, hence, that the conditions of Theorem 5 actually characterize $E(X \mid \mathscr{C})$.

\section{REFERENCES}

1. E. S. Andersen, and B. Jessen, Some limit theorems on set-functions, Mat.-Fys. Medd. Danske Vid. Selsk. 25 (1948).

2. H. D. Brunk, Conditional expectation given a $\sigma$-lattice and applications, Ann. Math. Statist. 36 (1965), 1339-1350.

3. H. D. Brunk, G. M. Ewing, and W. R. Utz, Minimizing integrals in certain classes of monotone functions, Pacific. J. Math. 7 (1957), 833-847.

4. P. R. Halmos, Measure Theory, Van Nostrand, 1964. 
5. S. Johansen, and J. Karush, On the semimartingale convergence theorem, Ann. Math. Statist. 37 (1966), 690-694.

Received April 4, 1966. This paper was prepared while the author was participating in a NATO Science Fellowship Programme.

UNiversity of California, Berkeley

AND

UNIVERSITY OF COPENHAGEN 


\section{PACIFIC JOURNAL OF MATHEMATICS}

\section{EDITORS}

H. SAMELSON

Stanford University

Stanford, California

J. P. JANS

University of Washington

Seattle, Washington 98105
J. DugundJI

University of Southern California

Los Angeles, California 90007

RICHARD ARENS

University of California

Los Angeles, California 90024

\section{ASSOCIATE EDITORS}

E. F. BECKENBACH

B. H. NeumanN

F WolF

K. YOSIDA

\section{SUPPORTING INSTITUTIONS}

UNIVERSITY OF BRITISH COLUMBIA

CALIFORNIA INSTITUTE OF TECHNOLOGY

UNIVERSITY OF CALIFORNIA

MONTANA STATE UNIVERSITY

UNIVERSITY OF NEVADA

NEW MEXICO STATE UNIVERSITY

OREGON STATE UNIVERSITY

UNIVERSITY OF OREGON

OSAKA UNIVERSITY

UNIVERSITY OF SOUTHERN CALIFORNIA
STANFORD UNIVERSITY

UNIVERSITY OF TOKYO

UNIVERSITY OF UTAH

WASHINGTON STATE UNIVERSITY

UNIVERSITY OF WASHINGTON

AMERICAN MATHEMATICAL SOCIETY CHEVRON RESEARCH CORPORATION TRW SYSTEMS

NAVAL ORDNANCE TEST STATION

Printed in Japan by International Academic Printing Co., Ltd., Tokyo Japan 


\section{Pacific Journal of Mathematics}

\section{Vol. 21, No. $1 \quad$ November, 1967}

Friedrich-Wilhelm Bauer, Der Hurewicz-Satz................... 1

D. W. Dubois, A note on David Harrison's theory of preprimes . ......... 15

Bert E. Fristedt, Sample function behavior of increasing processes with stationary, independent increments ..................... 21

Minoru Hasegawa, On the convergence of resolvents of operators....... 35

Søren Glud Johansen, The descriptive approach to the derivative of a set function with respect to a $\sigma$-lattice ....................... 49

John Frank Charles Kingman, Completely random measures ............ 59

Tilla Weinstein, Surfaces harmonically immersed in $E^{3} \ldots \ldots \ldots \ldots \ldots . . \ldots 9$

Hikosaburo Komatsu, Fractional powers of operators. II. Interpolation spaces ......................................... 89

Edward Milton Landesman, Hilbert-space methods in elliptic partial differential equations ...................................... 113

O. Carruth McGehee, Certain isomorphisms between quotients of a group algebra ........................................ 133

DeWayne Stanley Nymann, Dedekind groups .................. 153

Sidney Charles Port, Hitting times for transient stable processes ......... 161

Ralph Tyrrell Rockafellar, Duality and stability in extremum problems involving convex functions . ............................ 167

Philip C. Tonne, Power-series and Hausdorff matrices . . .............. 189 